\title{
New ways with aggregation
}

\section{An improved calculation with the classical equations of aggregation suggests new tasks for those who carry out computer simulations of these problems.}

THE fashion for the study of aggregation in all its forms (from the formation of smoke particles to the polymerization of chemicals) is readily explicable. Quite apart from the several practical applications in which a better understanding would be valuable, computer simulations of aggregation processes have opened up an intriguing line of enquiry, confirming among other things that many aggregating particles do indeed have fractal, or non-integral, dimension.

General interest in aggregation is not, of course, new, but goes back at least to Smoluchowski's work in the 1920s. But how to make a bridge between the recent numerical work and the now-classical but phenomenological equations for which Smoluchowski is best known? Two people from the University of Utrecht, P.G.J. van Dongen and M.H. Ernst, have now made a brave attempt (Phys. Rev. Lett. 54, 1396; 1985 ) which, among other things, has the virtue of suggesting where the computer people should next look.

The two approaches are as different as they could be. The computer simulations are typified by the model of T.A. Witten, which has been much elaborated since it first appeared in 1981. Particles are allowed to diffuse from the edges of some piece of lattice (square or 5 cubic, say), with instructions that they should stick to an aggregate grown from a single particle at the centre if they should happen to reach an immediately neighbouring position. Physically, the circumstances may be likened to those in which crystal growth is limited by the speed with which solute molecules can diffuse through a solution. Aggregates formed in this way do indeed have fractal dimensions. Recent elaborations of this simple model have allowed particles to shift their position after reaching the growth surface of an aggregate, or restrict occupancy of relatively inaccessible sites.

The Smoluchowski argument is more formal, and based on an enumeration of the ways in which aggregates of different sizes can stick together to form still larger aggregates. (The opposite process, disintegration, is supposed not to happen.) Aggregates containing $N$ elementary particles will be formed (to a first approximation) only from pairs of smaller aggregates whose combined size is $N$. The rate at which this happens will be proportional to the number (or concentration) of each possible pair of constituent sub-aggregates, that is to the product $c_{j} c_{k}$ where $j$ and $k$ add up to $N$. The snag is that the constant of proportionality will depend on the size of each member of the pair of subaggregates, so that the rate of formation of particles of size $N$ is something like $K(j, k) c_{j} c_{k}$ summed over all possible pairs of constituent sub-aggregates. (A factor of two enters if the sum is written as a sum over both indices $j$ and $k$.)

The rate at which aggregates of size $N$ disappear is similarly calculated, but now aggregation with a particle of any size will remove an aggregate from the $N$-sized cohort. The result of adding these two rates together algebraically is a simple-looking set of equations expressing the rate of change of the quantities $c_{j}$ which are nevertheless intractable (because they involve products of the variables, and are thus non-linear). But this, of course, is irrelevant because nothing is known about the coefficients $K(j, k)$. The hope that the computer simulations might throw some light on the nature of this function is one of the reasons why the subject has recently been fashionable.

Experiment, in the nature of things, can be of only limited help. Light-scattering from an aerosol or a colloid can, for example, provide information about the mean size of aggregating particles and some information about the size distribution. But the chance of defining the infinite set of coefficients $K(j, k)$ by the results of even the most refined experiment is plainly small. So the trick is to try to pin down the coefficients $K$, considered as a function of two particle sizes, from what scraps of information there may be. And a few generalizations are possible. In aggregating systems, it appears, the mean size does increase steadily with time. Experiments have also shown that in many kinds of aggregating systems, the evolving size-distribution is independent of the initial distribution when a sufficiently long time has passed.

Von Dongen and Ernst set out to generalize an earlier discussion of the problem (in 1966) by S.K. Friedlander. They restrict themselves to functions $K$ which assume the form $j^{p} k^{q}$ when the aggregates are large, which is one sign of how much more needs to be done in this field. There are reasons for supposing that the sum of the two constants $p$ and $q$ cannot exceed 2 .

Some of the results are nevertheless simple and striking. The formation of a gel from, say, a coagulating colloid requires that the sum of $p$ and $q$ should be greater than unity. Indeed, it turns out that this condition is also sufficient in the sense that if the criterion is satisfied, the system will inevitably gel. And there is much that can be said about the size distribution of particles in a gelling system, both before and after they have set.

The chief interest of the new calculations will however turn on what they have to say about the scaling behaviour of the sizedistribution function in all kinds of aggregating systems. Experiments as well as computer simulations suggest that the size distribution of particles in an aggregating system has a degree of uniformity in the sense that the variables $c_{j}$ (which change with time) can be related to the average aggregation size (say $s$ ) by an expression of the form $s^{-r} f(j / s)$ where $r$ is a constant and $f$ some function of a single variable. Von Dongen and Ernst are able to calculate more about the characteristics of such a function, and of its implications, than has previously been possible.

That there should be a simplication of this kind is really remarkable. What it implies is that, with the passage of time, an aggregating system will evolve in such a way that the size-distribution of the particles is similarly related to the mean particle size. Part of what van Dongen and Ernst succeed in doing is to calculate the properties of the unchanging distribution function, in practice by calculating its moments (the mean, variance and so on).

While the results obtained in this way are not inconsistent with what has been learned from computer simulation, there is clearly a great deal to be learned about the relationship between the simulations and the mathematical form of the coagulation function. One would have thought that there is now plenty of promise in attempts to simulate the aggregation process for a few of the special circumstances in which van Dongen and Ernst have shown the equations to be reasonably tractable.

A particularly valuable goal would be the definition of the exact form of the coagulation function when most aggregates are still small. The obvious difficulty is that the numerical programs so far used in these simulations are devoid of much in the way of the physics of what happens when substantial aggregates stick together. To what extent does rearrangement then become possible, for example? That is also a challenge for experimenters. May it be one of the unplanned benefits of the interest in nuclear winter?

John Maddox 